\title{
BIBSEARCH: A multifaceted research tool
}

\author{
BRIAL H. WATTS \\ New York University, New York, New York
}

\begin{abstract}
On-line information services are extremely powerful vehicles for obtaining large amounts of relevant information in a relatively short time. These services are limited, however, in their ability to further process the information they produce. BIBSEARCH is a local data base retrieval system that enables researchers to postprocess downloaded information, to combine this informaton with other similarly downloaded data, and to electronically rescan it at a later date. The user can also add notes, keywords, and so forth to these files. References can be manually entered and/or downloaded. Other BIBSEARCH features include support for: maintenance of an overlapping category filing system; extraction and listing of citations from manuscripts; automatic generation of sorted bibliographies; and the ability to test whether abstracts already exist within one's personal data base before downloading them.
\end{abstract}

Nowadays, almost any type of information one might require is available through on-line computer information services. In particular, electronic literature searches can be conducted by using a public on-line data base, such as DIALOG. Computer searching has a number of distinct advantages over manual systems. One major disadvantage of a manual search is that it is likely to be inefficient and incomplete. Computer-based searches, on the other hand, are much more efficient and are less likely than a manual system to contain gaps.

One reason for this increased efficiency is the sheer. speed of computers in performing letter sequence (strings of words) matching. The other reason is that computers can very easily and accurately perform Boolean decision operations on the indexed words of each article (which sometimes include every word of the abstract). These Boolean decision criteria can be combined in complex ways, enabling the researcher to conduct a search containing a minimum of false alarms and a maximum of hits. For example, in conducting a manual search, one can use the printed form of Psychological Abstracts and its associated keyword list to find all articles that have "Reading Ability" as a keyword; but if one were interested in a subpopulation of articles that also contains the keyword "School Age Children," one would have to search manually through all abstracts from the first list, excluding those not contained in the second list. Using a computer, this search can be done automatically with a single command and takes only a few seconds to execute. Of course, computer-based search requests can get much more complicated and can exclude, for example, articles published prior to specific dates or can select those that appear in specific journals.

Psychological Abstracts is available in a computerized form on a few on-line retrieval services, such as DIA-

I thank Doris Aaronson for helpful comments on an earlier draft of this paper. The author's mailing address is: Department of Psychology, New York University, 6 Washington Place, 8th floor, New York, NY 10003.
LOG, KNOWLEDGE INDEX, and BRS. This data base contains references and abstracts for more than 500,000 articles from over 1,000 sources, dating from 1969 to the present. Unfortunately, the on-line services are relatively expensive and may be rather wasteful if one finds oneself continually rescanning the data base for related articles. In addition, having captured the information from the on-line service, an individual can do little with it other than generate a hard copy printout and manually scan it.

When the number of retrieved abstracts becomes large (hundreds or thousands), the investigator's private data base becomes unmanageable unless there is a suitable computer program for manipulating and searching through it. BIBSEARCH ${ }^{1}$ was initially conceived as a tool for this purpose. It translates downloaded information into its own internal format, enabling local searches encompassing all the power of the on-line services to be performed at leisure by the researcher. Information can be added to the data base by the user (e.g., notes can be appended to the abstracts), and articles can be entered by hand if one does not want to download them from the on-line service. There are other benefits that BIBSEARCH provides: the automatic generation of sorted reference lists; the extraction of citations from documents; the ability to assist in the filing of articles; and the ability to create an electronic index to one's personal collection of journal volumes.

An emerging technology, namely that of laserdisk-based data (CD ROM), is adding to the capabilities of computer-based searching. It is now possible to store more than $500 \mathrm{MB}$ ( 500 million characters) of data on a single $51 \frac{1}{4}$ in. laser disk. Some data bases are now available to end users on compact disks that can be used with personal computers (see Waurzyniak, 1986, 1986, for a list of publishers of information on laser disks). The $\$ 5,000$ price tag on most of these data bases is currently beyond the reach of individuals, making them accessible only to large corporations or libraries. However, it is anticipated that costs will drop sharply within the next 
5 years as the user base expands to more than 10 times its current level (Waurzyniak, 1986).

In the meantime, researchers rely on the information provided by on-line information vendors, such as DIALOG and BRS. These services have at least three major problems: they are very expensive, averaging about $\$ 50$ per hour of connect time and about $30 \mathrm{c}$ per printed abstract ("International Comparative Price Guidè,"' 1986); they have no (or very limited) file manipulation, manual data entry, editing, or formatting capabilities; and the user must repeatedly pay for references retrieved in previous searches that are contained in subsequent searches. For these reasons, end-user local software (EULS) programs that attempt to address these problems are being developed for use on personal computers. These programs are, however, inadequate and/or costly (for reviews, see Faraone, 1986; Lundeen, 1986; Reneau, 1986; VidalArbona, 1986). After experiencing all of the above problems with on-line data bases, I decided 2 years ago to develop a EULS system, BIBSEARCH, which would be as efficient and powerful as the on-line systems, while maintaining the flexibility and user friendliness of a personal-computer-based system.

\section{FEATURES OF BIBSEARCH}

BIBSEARCH combines the features of EULS systems-file manipulation, editing, downloading, and report writing capabilities-with those typical of the public on-line systems, such as DIALOG and its European equivalent, QUESTEL. The on-line information systems typically have the following advantages over EULS systems: they employ a highly interactive and powerful command-based search request structure; they enable selection sets to be combined; and they employ efficient binary tree indexing methods (as opposed to the usual slow sequential searching of EULS systems) for the rapid retrieval of information from data bases that contain from hundreds of thousands to millions of references. In addition to the combination of features just mentioned, BIBSEARCH has a number of features not found in other information retrieval programs. These are listed below. See Figures 1 and 2 for a sample DIALOG session and its BIBSEARCH equivalent.

\section{Unique Features of BIBSEARCH}

Transformation program generator. The transform command enables users to transform data captured from on-line information services into the format supported by the BIBSEARCH system. Transformation programs are generated from user-specifiable input format specification (IFS) files. BIBSEARCH provides menu-selectable IFS files for the popular on-line services, DIALOG, KNOWLEDGE INDEX, BRS, and CAS (see Figure 3 for a listing of the DIALOG IFS file). For formats not explicitly supported by BIBSEARCH, the user can type in his/her own customized IFS file and add it to the IFS files used by BIBSEARCH.
The report generator. The built-in report generator is invoked by the print command. This command is normally used to generate annotated bibliographies or reference lists. The printing format can be chosen from a number of menu-selectable options. The print command produces a report-generation program from an output format specification (OFS) file, in a manner similar to the way "transform" interprets the IFS files mentioned above. System-supplied OFS files include American Psychological Association and American National Standards Institute standard formats. If the user wants to format output according to a format not explicitly provided by the system, he/she can create an OFS format specification file that will create any kind of output format desired. OFS and IFS files share an identical syntax (i.e., the user need only learn one format specification syntax to be able to create these files). See Figure 4 for an OFS description and its associated output listing.

Flagging paper citations. The cite command extracts citations from the body of a paper and searches the data base for matching references. Citations that contain nonstandard punctuation are listed with asterisks and a warning message. A list of matches, nonmatches, and ambiguous citations is provided, enabling reference lists to be automatically produced from the matching reference sets.

Verifying before downloading. The verify command is used to verify that references are not present within a private data base before the user downloads them from an on-line data base facility. Most on-line systems permit users to inspect short forms of references (which normally include titles) before printing out the entire reference. By checking the titles of local references against those within a short-form downloaded file, BIBSEARCH can indicate which references already exist within the data base. The user can then sign on to the on-line system and retrieve those articles of interest that are not contained in the local data base (see Figure 5 for a graphic description of this procedure). This method can result in considerable savings, particularly if one makes frequent use of on-line services and conducts searches within the same subject area.

Default truncation search mode. Most information retrieval programs have a facility for the specification of truncation (also known as wildcarding). The use of truncation enables the user to match words with common initial but varying final letters (e.g., computer and computers , or psychology and psychological). These words would be expressed as "computer?" and "psycholog?" using the "?" truncation character. The naive user, however, often is unaware of the significance, or even the existence, of wildcard facilities and may therefore often miss references that would have been retrieved using truncation. Even experienced users often forget about possible ending variations of words or phrases. For this reason, BIBSEARCH adopts truncation as the default mode for search commands, with "." overriding truncation. For example, "research." matches the word "research" but not "researching." Truncation, by default, serves to max- 
WELCOME TO KNOWLEDGE INDEX

Accounting atarting at $1: 12: 18$ EST
Date: Tue 25mar86

?B PSYC1

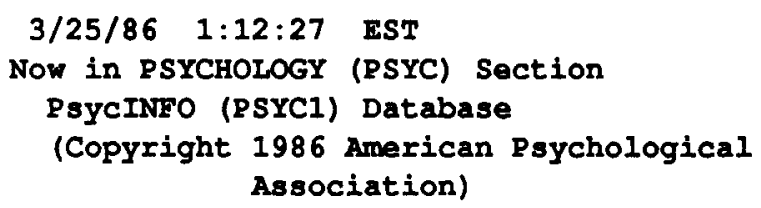

?E WORD PERCEPTION

187 WORD PERCEPTION

?E WORD SUPERIORITY

S2 62 WORD SUPERIORITY

?E ORTHOGRAPH? AND (RFDUND? OR STRUCTUR? OR REGULAR?)

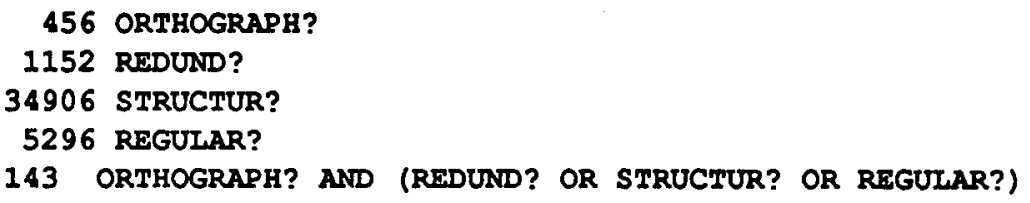

?F $S 1$ OR $S 2$ OR $S 3$

S4 272 S1 OR S2 OR S3

$? D S 4 / S / 1-272$

$4 / 5 / 1$

73-04065 Vol No: 73 Abstract No: 04065

The nature of phonological processing in oral reading: Evidence from surface dyslexia.

$4 / 5 / 2$

73-03711 Vol No: 73 Abstract No: 03711

Comparison of the gender-linked language effect and sex role stereotypes.

. . .

$4 / 5 / 272$

41-04542 Vol No: 41 Abstract No: 04542

WORD-FREQUENCY EETECT AND RESPONSE BIAS.

?IOGOEE

3/25/86 1:20:10 EST

Session Total: 0.134 Hours $\$ .3 .22$ User U45527

Figure 1. An example KNOWLEDGE INDEX session. Lines typed by the user are underlined for clarity.

imize hits, at the expense of raising the false alarm rate for retrievals. In addition to being friendlier for novice users, this strategy is preferable for smaller data bases and for local data base searching, where search costs are not a factor for consideration. In other words, a conser- vative approach to reference retrieval is usually preferable for local searching, whereas a more restrictive approach is required for large on-line data bases in order to minimize the amount of false alarms (useless downloaded information). In spite of this, it appears that all 


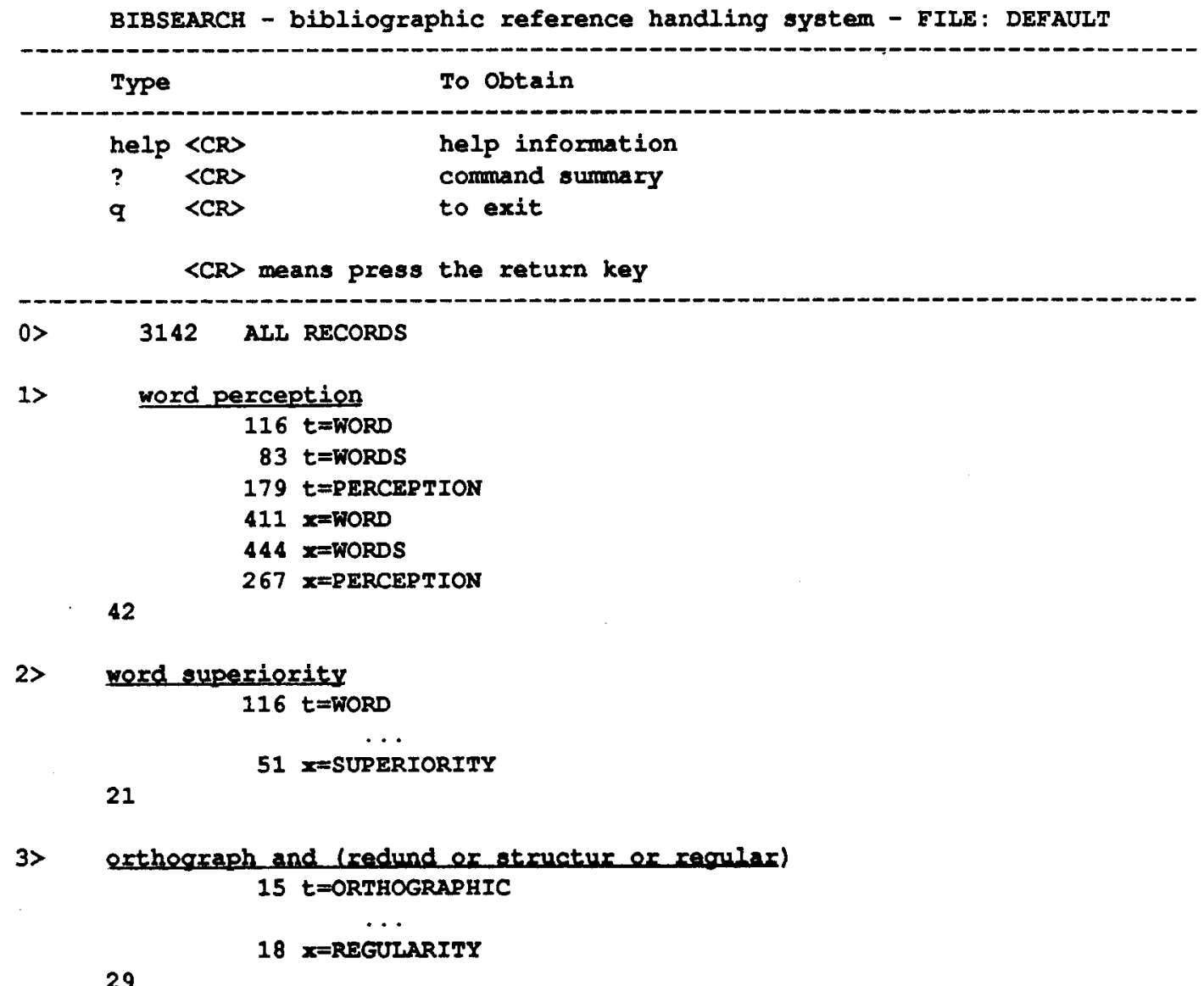

4) $\frac{1 \text { or } 2 \text { or } 3}{88}$

4) $\quad \mathrm{d}[2,3]$ at

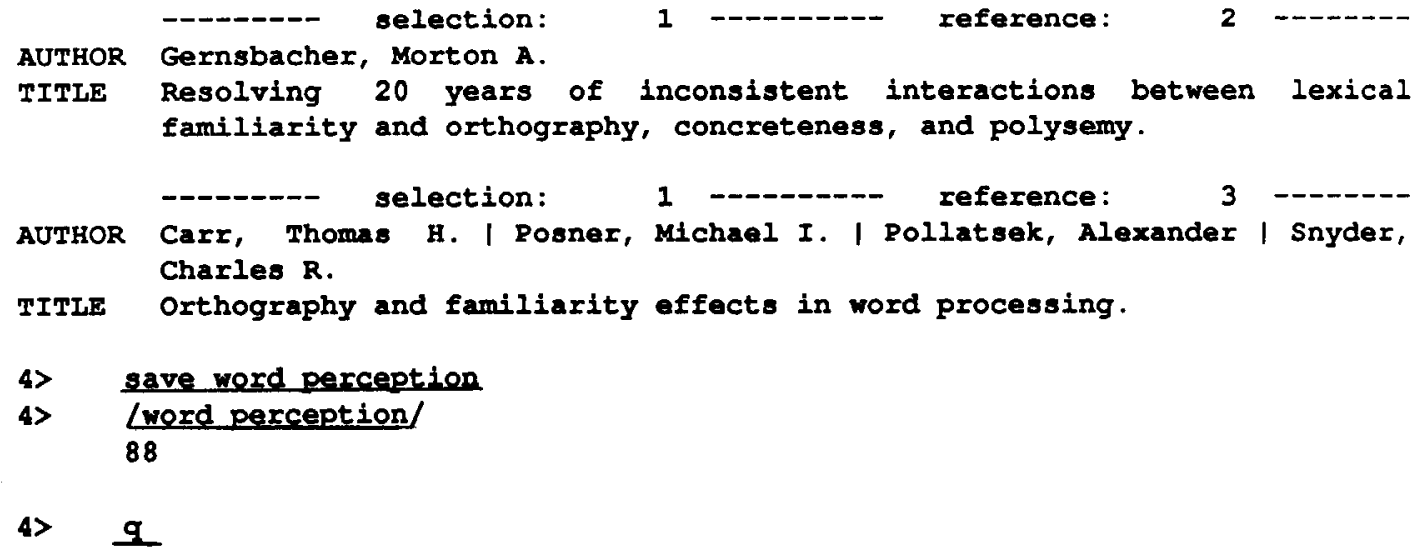

Figure 2. An example BIBSEARCH session. Lines typed by the user are underlined for clarity. Note the use of the save command, which saves pointers to the most recent set created in a named file (in this example, "word perception"). The references are extracted by typing the name of the saved file within slashes.

other EULS systems adopt the restrictive on-line searching strategy for local data base searches.

Within-phrase implicit and explicit truncation. Truncation can be used within phrases; for example, " $\mathrm{j}=$ $\mathrm{m} \& \mathrm{c}$ " will match Memory \& Cognition within the " $\mathrm{j}$ " (journal) field. Embedded words within phrases can also be omitted; for example, " $j=j$ e p" will match all of the Journal of Experimental Psychology journals. The explicit "?"' truncation character can be used to specify truncation at the start of a phrase; for example, " $k=$ ?learning" 


\section{A DIALOG INPUT FILE}

?D $s 2 / I / 9,10,11,13,27,28$

$2 / \mathrm{L} / 9$

72-09891 Vol No: 72 Abatract No: 09891

Alexia and the neural baste of reading.

Benson, D. Frank

U California, School of Kadicine, Los Angeles

Annale of Dyelexia 1984 Vol 34 3-13 ISSN: 07369387

Language: ENGLISH Document TYPe: JOURWAL ARTICLS

Three main types of acquired reading disturbance (alexia) have been described and accepted by cliniclans. Each features a group of distinct findings and each $\cdots$

Descriptore: ALEXIA, CERERRAL DOMINANCE, BRAIN DAMACE:

Identifiers: damage to different locatione within language-dominant

hemiephere of brain, types of alexia, conference preaentation

Section Headinge: 3290 . (PHYSICAL c PSYCHOSOMarIC DISORDERS)

...

\section{THE 'IFS' DESCRIPTION}

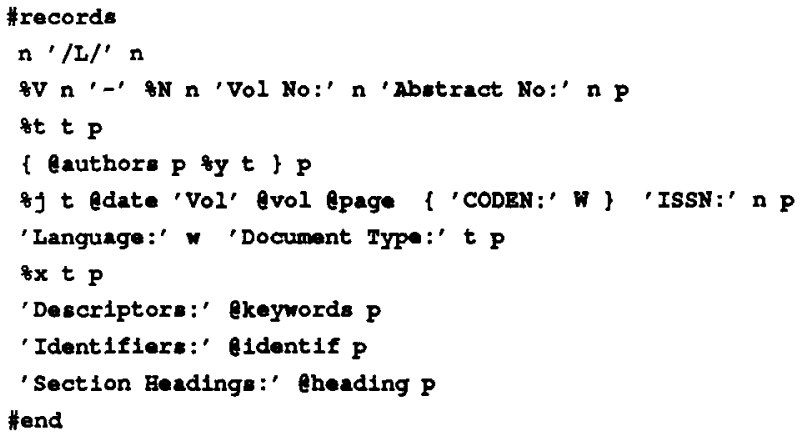

\section{THE 'TRANSFORMED' FILE}

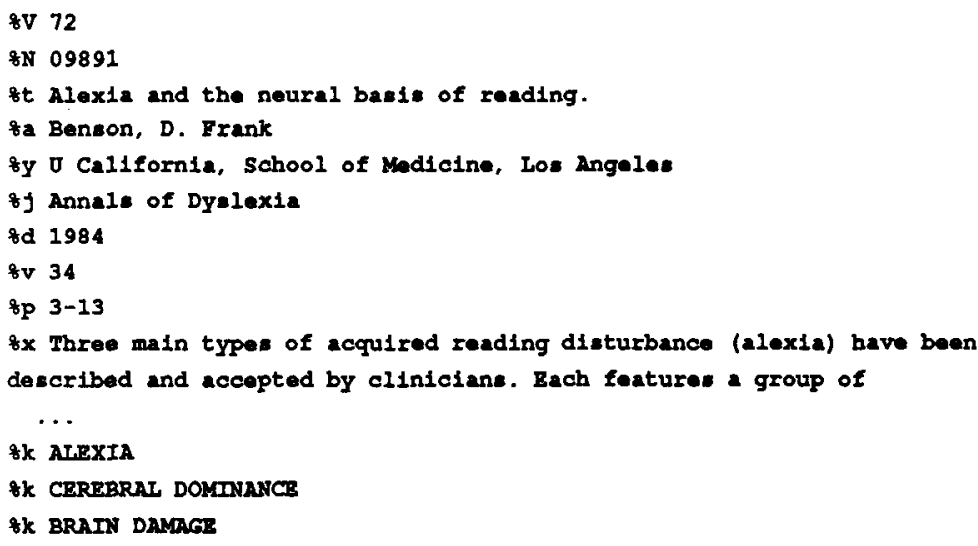

Figure 3. Transforming a DIALOG file into BIBSEARCH format. A portion of the DIALOG input file is shown in the top section. The middle section shows the main part of the IFS file, which specifies the input format and the information to be placed in specific data base fields. The "transform" program looks for input objects within the source file and places these (where specified by the sequence $\%<$ field $>$ ) within specific data base fields. Some standard input objects are numbers (n), free text (t), and paragraphs (p). Words beginning with @ are system-supplied macros for processing typical input objects. Optional input sequences are enclosed in braces. Part of the transformed file is shown in the bottom section. 


\section{AN 'OFS' DESCRIPTION}

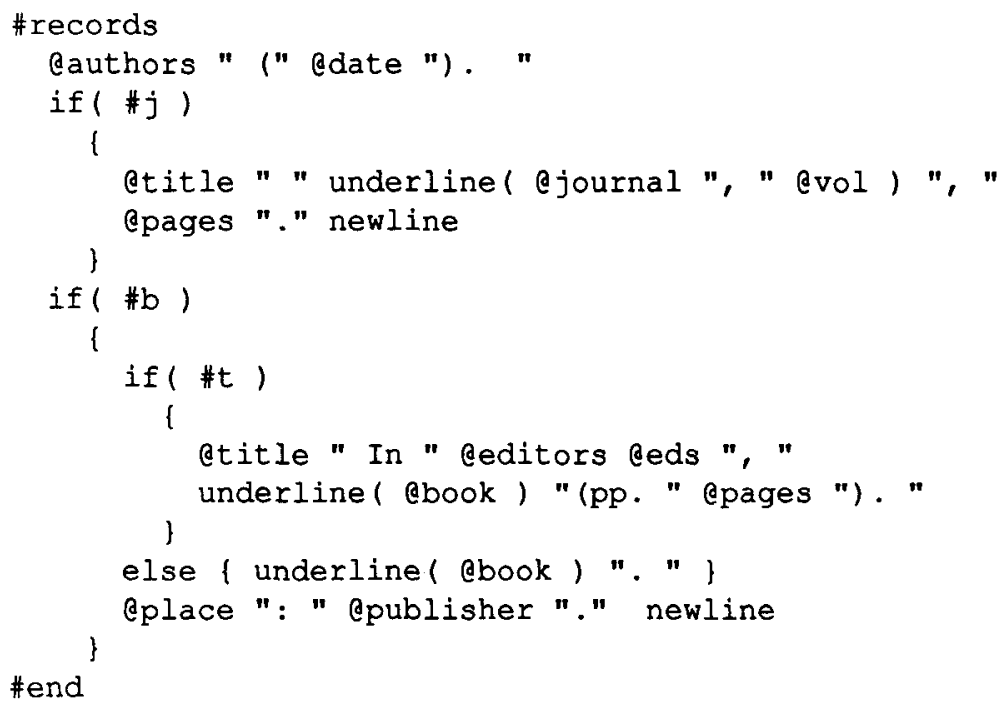

\section{A GENERATED REFERENCE LIST}

Aaronson, D. (1979). A cognitive approach to the study of language. In D. Aaronson, \& R. W. Rieber (Eds.), Psycholinguistic research (pp. 65-108). Hillsdale, NJ: Erlbaum.

Kaufman, L. (1974). Sight and mind. New York: Oxford University Press.

Sperling, G. (1986). A signal-to-noise theory of the effects of luminance on picture memory: Comment on Loftus. Journal of Experimental Psychology: General, 115, 189-192.

Figure 4. The automatic generaton of a sorted reference list. The top panel shows part of the OFS file, which produces a sorted bibliography using the American Psychological Association style. Words beginning with @ are system-supplied macros for the formatting of common field entries. Text within quotes is inserted in the output file. The expressions \#< field > produce the number of items (words or phrases within that field) for a particular reference. If there are no items, a zero value is returned; for example, \#j evaluates to zero if the journal field is missing.

will match both "paired associate learning" and "learning theory" within the " $k$ " (keyword) field of records within the data base.

Implicit search commands. To search for words within the current data base, the user merely types in the words to be searched for (i.e., the search command is implicit). Other commands are recognized by context. For example, if the user types "computer," the program responds by searching the data base for all words or phrases beginning with the letter sequence, "computer." Typing the display command " $\mathrm{d}$ at," however, causes the authors and titles ("at") of these articles to be displayed on the computer screen, rather than the sequence " $\mathrm{d}$ at" being searched for within the data base. In other words, if the program recognizes a command with the correct syntx, the command is executed; otherwise, the typed string is assumed to be a search request.
Combined sequential and indexed searching. EULS systems normally use sequential search methods to match records within the data base. The reasons for this approach are that sequential searching algorithms are easier to implement than alternative indexed searching methods; indexed files take up additional disk space; and the user has to reindex the data base each time it is updated when using indexed retrieval. The advantages of indexed retrieval are that large data bases can be scanned very rapidly and that alphabetized lists of field entries (such as authors, journals, or keywords) are naturally obtainable from the index lists. For on-line data bases, indexing is essential because the data bases are much too large to be searched sequentially. The inconvenience of reindexing is not felt by the user, because it is done by the vendor, and the user is not able to update the data base anyway. BIBSEARCH was designed to be used both for very large (e.g., CD 


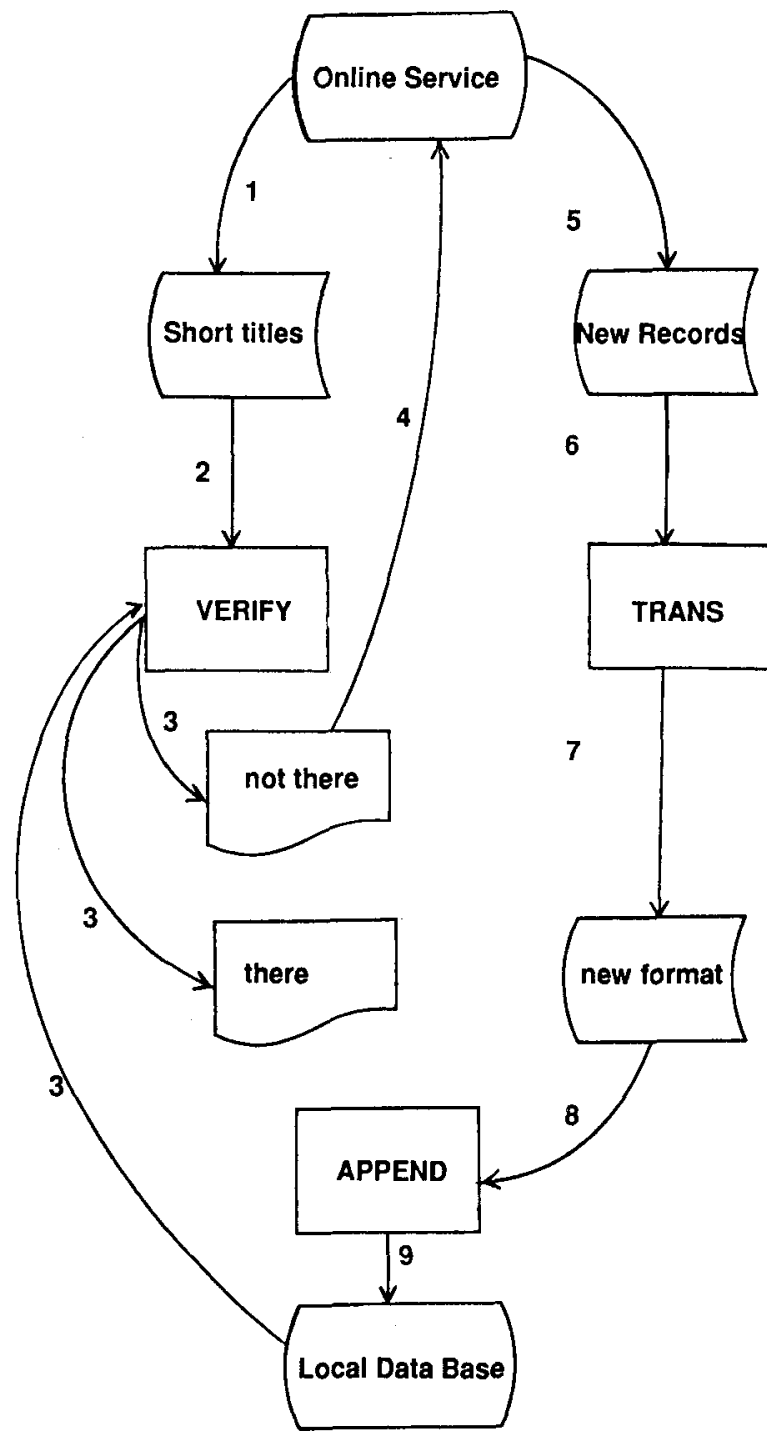

Figure 5. Using the verify command. Step 1: the user connects to an on-line information service, performs a search, and produces a list of short titles of matched articles. Steps 2 and 3: "verify" scans the titles list and produces a list of articles not found in the local data base. Step 4: the user connects to the on-line service once more and requests a printout of the missing abstracts. Steps 5-9: these new abstracts are transformed to BIBSEARCH format and appended to the local data base.

ROM based) local systems and for data bases of more modest sizes, consisting of, say, a few hundred references. To avoid forcing the user to reindex the data base each time it is updated, there are two modes of searching: sequential and indexed. The two modes can be combined within the same session, using an identical search syntax, except for a ":"' prefix that precedes commands for searches to be conducted in sequential mode. This means that fast indexed searches can be done using indexed mode, and the slower, more complete sequential mode can be used to match text in recent updated parts of the data base. The program also can automatically adopt sequential mode by default if the data base to be searched has not yet been indexed.
Virtual file support. Most people have difficulty in filing articles, because there is nearly always an overlap between potential filing categories. If all references have been entered (manually entered, downloaded, copied, or a combination of the three) into a BIBSEARCH data base, the articles can be filed in a filing cabinet according to first author, and subsets of articles can be defined within the computer data base by means of the BIBSEARCH save command. This command creates a computer file that consists of pointers to the selected articles (the set is created by using the normal BIBSEARCH search commands). One can think of these files as the computer analogue of a filing cabinet, with the important difference being that the categories defined by the computer files are potentially overlapping sets, whereas those of the filing cabinet are, of necessity, disjoint. By using this method of article filing, a user who is looking for articles within a particular category of interest merely runs BIBSEARCH: he/she types the appropriate set file name and inspects the references on the computer screen. Having found references of interest, the user physically locates the articles by displaying the author field and selecting the articles from the alphabetically arranged filing cabinet.

Index-aided manual entry. After data are manually entered into a BIBSEARCH text file, the indexes created when the data base is indexed can be used to make data entry of fields (e.g., author(s), journal, keywords, etc.) easier and more accurate. For example, when typing in the journal field of a reference, one might type " $\mathrm{j}$ e p." BIBSEARCH responds by listing the names of all matching journal names (in this example, all of the Journal of Experimental Psychology journals). The user then types the number that corresponds to the desired journal name, and the program responds by filling in the complete entry. This feature is useful for the following reasons: (1) It serves as a typing aid and reduces potential typing errors. (2) A check can be made as to the existence of keywords within the data base before they are added. For example, one can type "?LEARNING" in response to the keyword prompt; get a list of keyword phrases that contain "LEARNING"; and then select the appropriate one from the list, rather than having to guess which keyword to use. (3) In many cases, the user is likely to be unsure about the details of the full entry. For example, for the location field entry " $U$ Western Ontario, London, Canada," the user can type "u western ontario," and the program produces the full entry if there is at least one instance of "U Western Ontario, London, Canada" within the data base. (4) The user may be unsure of the form of the entry, for example, whether or not to type an ampersand in Memory \& Cognition. Typing "mem cog" is sufficient to produce the correct entry.

Text compression. The compress command is a data compression feature that enables the data base text file to be compressed to $25 \%$ of its original size. This feature is particularly useful for very large data bases (consisting of thousands of records). BIBSEARCH automatically converts compressed text to its uncompressed form be- 
fore printing. Other than initially typing the compress command, the usage of a compressed text data file is transparent to the user.

An on-line manual. The full text of the BIBSEARCH manual is accessible from within the program. Either the manual can be read by chapter and/or section via the help menu system, or information about commands can be directly obtained by typing "help < command $>$," where $<$ command $>$ is any of the BISEARCH system commands.

\section{DATA BASE CREATION}

There are a number of user alternatives for the creation of a BIBSEARCH data base. Many researchers already maintain a computer-based literature data base which they have either manually created or built up from downloaded data and augmented with personal notes. IFS files can be created to convert these data bases to the format required by BIBSEARCH. For those who do not have such data bases, references can be manually entered with the BIBSEARCH add command or downloaded from an on-line data base. For those who have used the UNIX Refer system, BIBSEARCH files are $100 \%$ compatible with the format dictated by the Refer program (Tuthill, 1983). In other words, data files that have been created according to this format can be used by BIBSEARCH without modification.

\section{DATA BASE COSTS}

Downloading references from an on-line service can be expensive, depending on the service used, the transmission speed, and, of course, the amount of data required. The cheapest service is an after-hours service called Knowledge Index, provided by DIALOG. This service currently includes about 30 popular data bases, including Psycinfo, the computerized version of Psychological Abstracts. The charge is $\$ 24$ per hour, and there are no hidden charges, such as monthly minimums or on-line printing charges. Moreover, there are no surcharges for high transmission speeds, and the service supports connection at 2400 baud. This means that it is possible to download data from Knowledge Index for about $5 \mathfrak{c}$ per reference (including abstracts). This rate is very attractive when one considers that a complete set of 10 years of abstracts from 10 journals can be downloaded at a cost of about $\$ 500$ or less. Updates could be done biannually at a minimal cost, and users could supplement this big central data base with subject-specific abstracts within their field of specialization. In this manner, a psychol- ogy department could be served by a data base downloaded in this way, from which individuals could siphon references for their own usage, personalize them by adding notes, and so forth. Another very attractive possibility is that of the creation of a personal electronic index to one's private collection of journals. Using one of the on-line services, it is possible to download abstracts for specific volumes of specific journals. If the researcher downloads all of the abstracts for his/her private collection of journals, the data file can be used (with the assistance of BIBSEARCH) as a powerful electronic index to all of the articles within these journals.

\section{AVAILABILITY}

A demonstration disk of BIBSEARCH is available at cost of $\$ 5$ (for postage and disk) by writing to the author. The demonstration disk is a limited implementation of the BIBSEARCH system: the number of records is limited to 100 , and the index, compress, and transform commands are not included. ${ }^{2}$ The demonstration package can be used for the maintenance of relatively small local data bases. For those who are interested in experimenting with the deomonstration system and possibly upgrading at a later stage, data files created with the demonstration package are totally compatible with the full implementation system.

\section{REFERENCES}

Faraone, M. (1986). REF-11: A database for references. Online Review, 10, 163-164.

International comparative price guide to databases online. (1986). Online Review, 10, 249-260.

LUNDEEN, G. (1986). CAIRS: Local detabase information storage and retrieval. Online Review, 10, 159-161.

RENEAU, F. W. (1986). Searchware: for online access by novice users. Online Review, 10, 35-36.

TuthILL, B. (1983). Refer-A Bibliography system. UNIX User's Manual Supplementary Documents. Berkeley: University of California-Berkeley, Department of Science.

VIDAl-ArBona, C. (1986). FINDER: Software for text retrieval-A review. Database, 9, 43-52.

WaurzYniak, P. (1986, December 8). Optical discs. Info World, 8, $51-55$

\section{NOTES}

1. BIBSEARCH runs under UNIX (or XENIX) and has recently been ported to MSDOS, enabling it to run on standard IBM PCs (or compatibles).

2. The cost of the full system is $\$ 200$, with a $50 \%$ discount for students. Site licenses, with which unlimited copies of the program can be made, or multiple users can access the program, are available at a cost of $\$ 600$. 